\title{
Correction to: Stress Fractures of the Pelvis and Sacrum
}

Burak Altintas, Timothy L. Miller, and Mary Lloyd Ireland

Correction to: Chapter 15 in: T. L. Miller, C. C. Kaeding (eds.), Stress Fractures in Athletes, https://doi.org/10.1007/978-3-030-46919-1_15

The spelling of the author name was inadvertently published as Burak Alintas in the Table of Contents and Chapter 15.

This has now been amended throughout the book as Burak Altintas.

The updated online version of the original chapter can be found at https://doi.org/10.1007/978-3-030-46919-1_15 